sein. Die Geschichte der Medicin zeigt nur zu oft, dass sich die best ausgedachte Therapie als unbrauchbar und gar manches auf einer unrichtigen Theorie basirte Mittel als ausgezeichnet erwiesen hat. Theoretische und aprioristische Erwägungen können bei der Wahl einer Behandlung einigermaassen leiten, das endgiltige Urtheil muss die klinische Empirie sprechen.

Wenn wir uns nun heute ein Urtheil über den jetzigen Standpunkt der Gichtbehandlung bilden wollen, so können wir dies nur, sobald wir bei jedem Punkte auch die Erfahrungen der älteren Zeit zu Rathe ziehen. Und auch dann können wir, wie es sich zeigen wird, nicht auf alle Fragen eine befriedigende Antwort ertheilen

Bei der Behandlung der Gicht kommen von Alters her in

\section{Altes und Neues über die Behandlung der Gicht.}

Ein historisch-kritisches Referat.

Von Privatdocent Dr. Maximilian Sternberg in Wien.

In den letzten zehn Jahren hat sich die wissenschaftlicle Forscliung in ausgedehntem Maasse mit der Gicht beschäftigt. Insbesondere haben die Fortschritte der Chemie dazu beigetragen, welche uns zuerst neue und bessere Bestimmungsmethoden der Harnsäure und die synthetische Darstellung dieser Substanz gelehrt, dann den Zusammenhang der Harnsäure mit zwei sehr wichtigen Gruppen von Substanzen, einerseits den Nucleïnkörpern, andererseits den Xanthin- oder Alloxurbasen aufgedeckt haben. Dadurch haben wir ganz neue, noch vor kurzem ungeahnte Aufschlüsse über die Schicksale gewonnen, welche alle diese chemischen Verbindungen im menschlichen Körper erleiden, und ganz neue Einblicke in ihre Bedeutung für den Organismus. Hand in Hand mit der chemischen Arbeit haben sich die Kliniker bestrebt, die neue Erkenntniss für die Vertiefung unserer Einsicht in das Wesen der Gicht und der harnsauren Diathese überhaupt nutzbar zu machen und neue Gesichtspunkte für die Behandlung zu gewinnen. Dies war wieder der Ausgangspunkt für eifrige Bestrebungen der Chemiker, das seit Jahrhunderten, ja seit Jahrtausenden vergebens gesuchte "sichere Heilmittel " der Gicht endlich einmal zu finden.

So ist in den letzten Jahren die Litteratur ïber Gicht zu einer fast unübersehbaren Masse angeschwollen, mit ihr die Zahl der gegen Gicht empfohlenen Curmethoden und Heilmittel. Natürlich wächst in unserer Zeit der Reclame auch die Kraft, mit welcher Methoden und Mittel angepriesen werden, mehr und mehr. Klagte schon Trousseau über den Inseratentheil der medicinischen Zeitschriften, so wird heute der Arzt mit Annoncen und Broschüren über Gichtmittel geradezu überschwemmt

Umsomehr wird es dem Praktiker zur Pflicht, sich nüchtern und ruhig zu fragen, welche Fortschritte wir in der Behandlung der Gicht und der uratischen Diathese gemacht haben, welche alten Meinungen etwa auf Grund der neu gewonnenen Erkenntniss aufzugeben, welche neuen Verfahren, welche neuen Mittel aufzunehmen seien.

Die Beantwortung dieser Frage stösst auf ein eigenthümliches Hinderniss. Die Gicht ist eine der chronischsten Krankheiten, die wir kennen. Es giebt sehr viele Gichtleidende, die trotz aller Schmerzen und Beschwerden, mit verkrümmten und kaun brauchbaren Gliedern ein hohes Alter erreichen. Will der Arzt sich über den Werth einer Behandlungsmethode dieser Krankheit ein Urtheil bilden, so muss er nicht bloss die Einwirkung der Behandlung auf den Verlauf der Gichtanfälle, der Nierensteinkoliken u. s. w. kennen, also seinen Kranken Jahre lang verfolgt haben, er muss auch die sehr wichtige Frage zu beantworten in der Lage sein, ob die Behandlungsmethode ihn vor der bei der Gicht so häufigen und besonders gefürchteten Erkrankung der inneren Organe geschützt, ob sie diese verzögert oder etwa gar befördert habe. Mit anderen Worten, er muss nach Jahr und Tag die Section seines Kranken machen, das heisst, der Arzt muss den Gichtkranken überleben. Nur dann ist es wirklich möglich, ein Urtheil abzugeben, was genützt, was geschadet hat, was zu empfehlen sei, was künftig besser unterlassen werde. Da nun die allerwenigsten Aerzte das Glück haben, wie Morgagni im 80. Lebensjahre bei voller Geistesfrische die Summe aus den Erfahrungen ihres Lebens zu ziehen, so ist die Schwierigkeit begreiflich, zu einem Urtheil über Gichttherapie zu gelangen.

Darauf beruht es, dass wir über den Jahrhunderte alten Streit, ob gewisse Behandlungsmethoden, welche die Anfälle mildern, zugleich das Aufteten von Erkrankungen der inneren Organe bei Gicht "Arthritis interna“, "Arthritis anomala", "Arthritis retropulsa", "Gichtmetastasen" ("Gichtmetaschematisinen" Hufeland) - befördern und so das Leben verkürzen, noch heute nicht zu entscheiden vermögen. Nur die fortlaufende Arbeit und Beobachtung mehrerer aufeinanderfolgenden Generationen von Aerzten wird dies thun können.

Bei der Beurtheilung des Erfolges einer Behandlung darf uns nur dieser Erfolg, nicht aber irgend eine theoretische Vorstellung über das Wesen der Krankheit oder deren Chemismus maassgebend
Betracht: Diät, Alkalien, Specifica, physikalische Heilmethoden.

Man sollte glauben, dass in betreff der Diät die Erfahrung der Jahrtausende eine Einigung über die wichtigsten Gesichtspunkte erzielt haben könnte. Dem ist aber nicht so. Nur die schon von Hippokrates gegebene Regel ist allgemein anerkannt: Mässigkeit; wenige Speisen; einfache Zubereitung. Damit kommen wir aber nicht aus. Vier Fragen sind es hauptsächlich, die der Praktiker sich stellen muss, wenn sie nicht schon vom Kranken an ihn gerichtet werden: Ist Fleischnahrung oder Kohlehydratkost vorzuziehen? Ist Milch empfehlenswerth? Wie steht es mit dem Genusse saurer Speisen? Wie mit dem des Alkohols?

Die alte Erfahrung schien es festgestellt zu haben, dass Fleisch nicht allzu reichlich zı gestatten, dafür mehr Vegetabilien in die Diät einzuführen seien.

Wollaston hat im Anfange des Jahrhunderts die streng vegetarianische Diät empfohlen, wofür neuestens wieder Haig eilltritt. Dieser hat an sich selbst seit Jahren Studien gemacht, da er an uratischer Diathese leidet, sowie an vielen Uratikern und Gichtkranken. In der vegetarianischen Diät sieht er das sicherste und zugleich einzige Heilmittel. Das Fleisch ist durch Caseïn und Leguminose $\mathrm{zu}$ ersetzen. Auch G. Rosenfeld empfiehlt Verminderung der Fleischnahrung und theilweisen Ersatz des Fleisches durch Caseïnnatrium, Pepton und Aleuronat.

Ebstein befürchtet aber von reichlicher Kohlehydratzufuhr Verdauungsstörungen, wie $L 0 b b$, welcher schon im vorigen Jahrhundert die vegetarianische Diät bei Gicht verwendet hat und sie nicht als unbedingt empfehlenswerth betrachtet. Ebstein empfiellit vielmehr - viel Fett, namentlich Butter.

Ganz andere Ansicht vertreten - zwar von verschiedenen theoretischell. Standpunkten ausgehend, aber zu denselben Folg'erungen gelangend - v. Mering und E. Pfeiffer. Beide einpfehlen reichliche Fleischnahrung. Hierüber wurde in deu letzten Jahren manche Discussion geführt. Ebstein, C. Mordhorst, v. Noorden haben sich entschieden gegen diese Diät ausgesprochen.

Welche Fleischsorten darf man geben, welche nicht? Bis vor kurzem hatte man über die Unterschiede der Fleischsorten nur ganz nebelhafte Ansichten. Man begnügte sich mit der alten Unterscheidung von "schwarzem" und "weissem Fleisch" und hatte alt überlieferte, aber sehr zweifelhafte Indicationen für deren Verordnung. Bezüglich der anderen Theile: Leber, Bries, Niere und dergleichen entschieden alte unbegründete Vorurtheile oder - der persőnliche Geschmack des Arztes.

Seit Gaucher in den sogenannten Extractivstoffen des Fleisches Nierengifte erkannt und die Fleischbrühe bei Nephritis verboten lıat, müssen wir auch für die Gichtdiät die entsprechenden Folgerungen ziehen. Wir werden mit $\mathrm{Kolis} c h$ die Fleischbrühe untersagen, das Fleisch im gekochten Zustande - wie in Oesterreich üblich - dem gebratenen vorziehen. Allerdings ist auch diese Erkenntniss nicht so ganz neu, wie man glauben könnte: Scudamore gestattete schon im Anfange unseres Jahrhunderts den Genuss der Suppe nur in kleinen Mengen und verbot die kräftigen Suppen.

Durch die Arbeiten von Weintraud, Umber, H. Strauss u. a. haben wir die Thatsache kennen gelernt, dass nucleïnreiche Theile, wie Thymus und Leber, die Harnsäureausscheidung erheblich vermehren. Bei Neigung zur Concrementbildung in den Harnwegen wird man daher diese Theile, sowie das Fleisch junger Thiere - trotzdem es "weiss" ist - verbieten, ebenso junge Pflanzentheile, wie die Spargel, welche übrigens schon Boerhave untersagte. Ob aber die Beschränkung dieses Genusses bei der eigentlichen Gelenkgicht empfehlenswerth und nöthig ist, wird nach dem jetzigen Standpunkte unserer chemischen Kenntnisse von den verschiedenen Forschern noch verschieden beantwortet.

Sehr' alt ist die Frage der Milchdiät. Ueber ihre Zweckmässigkeit können theoretische und praktische Gründe entscheiden. Die chemische Unter'suchung zeigt, dass Milch die Xanthinbasen im Harn meist ein wenig steigert, die Harnsäure vermindert. Daraus können wir noch nicht ganz sichere Schlüsse ableiten. Die praktischen Erfahrungen der Kliniker widersprechen sich. Die alten Aerzte, Sydenham, Fr. Hoffniann, Boerliave, 
van Swieten haben sicl reservirt verhalten. Bei Durchfülurnng der absoluten Milchdiät bleibt der Kranke voll Anfällen verschont, allf die Dauer hält er es aber nicht aus. Viele vertragen die Milch in grösserer Menge iiberhaupt nicht. Aehnlich haben sich von neneren Ebstein und $\mathrm{Pfe}$ if $\mathrm{fer}$ alsgesprochen. In den letzten Jahren ist man der Milch wieder freundlicher gesinnt: Klemperer, Kolisch, Laquer, alles jüngere Schule, empfehlen den Milchgenuss warm. Namentlich die Gärtner'sche „Fettmilch"- wird gerühmt. Der Praktiker wird wohl gut thun, sorgfältig zu individualisiren.

Die sauren Früchte lnd sauren Speisen findet man in vielen Diätvorschriften bis in die allerletzten Tage, z. B. bei Beissel, streng untersagt. Andere Autoren, gerade sehr erfahrene Praktiker, gestatten oder empfehlen sie dagegen lebhaft. Es ist in der That auch nicht recht einzusehen, weshalb die Pflanzensäuren und pflanzensauren Salze zu fürchten wären, da sie ja im Körper zu Kohlensäure verbrannt und ausgeathmet, bei Gegenwart von Alkalimetallen aber zu kohlensauren Salzen umgesetzt werden, die seit uralter Zeit als Heilmittel der Gicht gelten.

Die Durchsicht der alten Schriftsteller ïber Gicht zeigt nun in der That, dass das Verbot der Pflanzensäuren auf alten, sonderbaren, humoralpathologischen Vorurtheilen beruht. Schon im 17. Jahrhundert gab es eine Theorie der "Säurevergiftung des Organismus". 1) Da beim Eintritt des Gichtparoxysmus nicht selten erbrochen wird und das Erbrochene natürlich fast immer sauer reagirt, sah man darin einen deutlichen Beweis der Ueberladung des Körpers mit Säuren und folgerte, dass die Zufuhr der Säuren in jeder Form zu verbieten sei. Obwohl nun längst die Ursache der sauren Reaction des Magensaftes aufgeklärt ist, obwohl schon längst die Schicksale der Pflanzensäuren im menschlichen Körper ermittelt sind, wird das Verbot der sauren Früchte noch immer von vielen als diätetisches Dogma weitergeschleppt. Einsichtsvolle Autoren, wie Ebstein und manche Andere, empfehlen freilich die sauren Früchte und die Curen mit Erdbeeren oder Kirschen. Die Citronensäure, welche vielfach angepriesen wird, hat nach den Untersuchungen von Haussmann allerdigs nur wenig Eillfluss anf den Säuregehalt des Harns. (Sie muss mit Alkalien verbunden gereicht werden. Klemperer.) Wir reihen hier zweckmässig die Empfehlung der Milchsäure von Bérenger-Ferand an, in Tagesgaben zu 2,0 einige Wochen hindurch gereicht. Da die milchsauren Salze im Körper zul kohlensauren verbrannt werden, ist vom theoretischen Standpunkte gegen einen Versuch mit einer solchen Behandlung, die nicht sehr sympathisch anfgenommen wurde, nichts einzuwenden. Entscheiden muss natïrlich, wie schon gesagt, einzig und allein die klinische Erfahrung.

Bezüglich des Alkohols sind die dentschen Antoritäten jetzt einig, dass er ganz zu verbieten sei. Franzosel und Engländer sind weniger streng, vielleicht weil sie sich in Bezug auf den Erfolg des Verbotes weniger Illusionen machen. Man findet bei ihnen eingehende Besprechungen der gestatteten und der verbotenen alkoholischen Getränke. Gesicherte Thatsachen, auf Grund deren man eine solche Unterscheidung machen kann, sind allerdings kaum zu finden. Es ist hier, wie leidel noch so vielfach in der Krankendiätetik, gar vieles auf rein subjectivem Ermessen begrïndet. Daher ist wohl nicht darauf einzugehen, dass Proust Obstmost verbietet, Moselwein gestattet, andere den Champagner verbieten etc.

Ein Punkt aus der Diätetik sei noch besprochen, das Kochsalz. Die alten Aerzte, so Fr. Hoffmann, verboten die stark gesalzenen Speisen. Mendelsohn hat unlängst gefunden, dass die Harnsäure und harnsaures Natron aus Lösungen sehr leicht gefällt, „ausgesalzt" werden, sobald man Chlornatrium oder Chlorlithinm zusetzt. Er hält es für möglich, dass diese Beobachtung praktische Bedeutung gewinne. Dies scheint nun nach einer heute vergessenen Beobachtung von Drew wirklich der Fall zu sein. Drew gelangte auf Gsund populärer Annahmen und theoretischer Ueberlegnng zu der Ueberzeugung, dass Kochsalz scliädlich sei, und sah bei absoluter Enthaltung von dieser Substanz in den Speisen wirklich die gichtischen Ablagerungen schwinden.

Von den Alkalien kommen seit alten Zeiten drei Gruppen in Betracht, die kohlensauren Salze des Natriums, Kaliums und Lithiums die Verbindungen der Erdmetalle Calcium und Magnesinm und die Ammoniakverbindungen.

Die kohlensauren Alkalien sind bekanntlich von Fr. Hoffmann, dem genialen Arzte und Chemiker, in die wissenschaftliche Gichttherapie eingefïhrt worden. Er gab sie besonders als Mineralwässer: Selters, Schwalbach u. s. w. In schweren Formen hielt er Karlsbad für unbedingt nöthig. Wenn hente von mancher Seite die Wirksamkeit der natürlichen Mineralwässer skeptisch betrachtet wird, darf man nicht vergessen, dass sie früher in solchen Mengen

1) Es ist nicht uninteressant, dass man diese Annahme schon damals durch Alkalescenzbestimmung im Blutselum zu prüen bestrebt war. Colbatch wollte gefunden haben, dass die Alkalescenz bei Gicht vermehrt sei und sich zu der des Gesunden wie $6-5^{1 / 2}: 4$ verhalte. getrunken wurden, dass eine wirksame Answaschnug des Körpers stattfinden konnte (bis zu 50 Gläsern täglich, ja mehr).

In den Arzneischatz der deutschen Aerzte ist seitdem die Alkalitherapie unangezweifelt aufgenommen worden. Nur die Formen wechseln von Zeit zu Zeit. Van Swieten empfahl den "Liquor nitri fixi", d. i. an der Luft zerflossenes kohlensaures Kalinm. Ferner eine Mischung von Alkalien mit pflanzensauren Salzen: Die Asche der Summitates Genistae mit Rheinwein infundirt. In neuester Zeit ist ein ähnlich zusammengesetztes Präparat unter dem tönenden Namen des "Uricedin" in den Handel gebracht worden, im wesentlichen eine Mischung von citronellsauren Salzen und Alkalien in der Form von Granulis.

Die verwendeten Mineralwässer wechseln von Zeit zu Zeit mit der wissenschaftlichen Mode und - der Kapitalkraft der Brunnenbesitzer, beziehungsweise deren Geschicklichkeit in der Reklame.

Die Entdeckung, dass harnsaures Lithium verhältnissmässig leichter löslich sei, hat alf Garrod's Empfehlung eine Zeit lang den Lithiumsalzen grossen Ruf verschafft, und es bemühten sich alle Brunnenverwaltungen, in ihren Quellen Lithium nach weisen zul lassen. Heute ist ziemlich allgemein erkannt, dass die Lithiumsalze vom Magen meist nicht gut vertragen werden. Anch wissen wir, dass sich die Harnsäıre, mit einer Lösung von mehreren kohlensauren Salzen zusammengebracht, nach ganz bestimmten Gesetzen, entsprechend dem relativen Mengenverhältniss der einzelnen Salze und den thermo-chemischen Beziehungen, mit den Metalloxyden verbindet, dass also bei einem kleinen Lithium- und bei einem grösseren Natriumgehalte eines Mineralwassers nur ein kleiner Theil Harnsäure an Lithium, aber ein grosser Theil an Natrium gebunden wird, dass also der geringe Lithiumgehalt einer Qnelle nur eine recht nebensächliche Rolle spielen kann. Trotzdem - in der Medicin wird eben eine einmal aufgenommene Meinung gar leicht zum Dogma - gilt das Lithium noch heute vielfach als Maassstab der Wirksamkeit eines alkalischen Mineralwassers.

In manchen Curorten, welche kein alkalisches Wasser besitzen, in denen aber doch die Gicht behandelt werden sollte, liess man fremde alkalische Sänerlinge trinken oder setzte dem Brunnen kohlensaures Natron zll. So ist z.B., wie der biedere Z ü ckert 1768 berichtet, in Wiesbaden der Gebrauch von Selters oder Schwalbacher eine ehrwïrdige Curtradition. Es ist vom ärztlichen Standpunkte dagegen gewiss nichts einzuwenden. Eher schon gegen den Zusatz von kohlensaurem Natron. Nicht jedes Mineralwasser ist dazu geeignet; in vielen finden chemische Umsetzungen zwischen den ursprünglichen Salzen des Brunnens und dem beigefügten statt. Dadurch fallen unlösliche Verbindungen aus, das Wasser muss durch Absetzenlassen in Bassins geklärt werden und bildet dabei als alkalisch reagirende Flüssigkeit eine gute Nührlösung für Bacterien, welche auch reicllich darin wuchern. So ist leider das seit einigen Jahren in den Handel gebrachte "Wiesbadener Gichtwasser" beschaften, welches aus dem "Kochbrinnen" durch Zusatz von kohlensaurem Natron und Kohlensäure erzeugt wird.

In Frankreich hat es viel länger gebraucht, bis man Alkalien bei Gicht anzluwenden wagte. 1835 machte Petit, Badearzt in Vichy, die ersten Versuche mit Anwendung des Vichy-Wassers bei Gicht unter allgemeiner Opposition. Laien und Aerzte waren noch lange in grosser Aufregung über diesen "kühnen Versuch". Noch am 25. Juni 1843 wurde der Ackerbauminister in der Deputirtenkammer interpellirt, ob eine Heilwirkung von Vichy bei Gicht officiell constatirt worden sei. Trousseau befürchtete sehr, dass durch Gebrauch von Alkalien die Entstehung der inneren Gicht hervorgerufen oder beschleunigt werden könnte.

Der Gebrauch des kohlensauren Kalkes ist uralt. Schon Dioscorides empfiehlt Korallen bei Gicht. Fr. Hoffmann verordnete bei Harngries und Nierensteinkolik gern den längeren Gebrauch der Lapides cancrorum, z. B. in folgendem Pulver, das allch heute ganz verwendbar erscheint:

Rp. Radic. pimpinellae nigr.

irid. florent.

liquirit.

Lapid. cancror. aa 25,0

Ol. macid. gtt. VI.

MDS. 2 Mal wöchentlich 4,0 zu nehmen.

Selbst in populären medicinischen Werken älterer Zeit, so im „LeibMedicus der Studenten" von Abelius, werden die Krebsaugen bei Steinbeschwerden und Podagra empfohlen. Das scheint in England unbekannt geblieben zul sein, denn das Geheimmittel gegen Harngries und Blasenstein, welches 1740 all Beschluss des Parlaments um 10000 Goldstiicke angekanft wurde, bestand aus venetianischer Seife, gebrannten Eierschalen und Schneckenhäusern. H in feland verordnete den kohlensauren Kalk bei Gicht und empfahl ihn, von dem Zusammenhange zwischen Gicht und Diabetes überzengt, aucl beim Diabetes. Seitdem ist der kohlensaure Kalk fast verschollen. Nur in den kalkhaltigen Mineralwässern (Fachingen, Wildungen) 
wurde er verwendet. In der allerletzten Zeit liaben $v$. Noorde ll und sein Assistent J. Strauss unser Mittel wieder zur Beliandlung der harnsauren Concremente empfohlen. Es ertheilt dem Harn in hohem Grade harnsäurelősende Eigenschaften, ohne ihn alkalisch zu machen. Bei dessen Verabreichung wird nämlich ein grösserer Antheil der Phosphorsäure im Darm ansgeschieden, so dass die Menge des Dinatriumphosphats im Harn vermehrt wird. Mordhorst fürclitet zwar, dass die Kalkbehandlung zur Entstehung von Arteriosklerose und zur Bildung von Kalkconcrementen im Harn führen könne. Nach v. Noorden besteht aber die Gefahr einer Bildung von Steinen aus kohlensaurem Kalk in den Harnwegen nicht, weil die saure Reaction des Harns erhalten bleibt.

Nach den mitgetheilten alten Erfahrungen ist die Kalkbehandlung gewiss empirisch berechtigt. Sie hat aber noch eine andere Gefahr, welche man ganz vergessen $\mathrm{zu}$ haben scheint: die Bildung von Kalkconcrementen im Darm. Solche sind in der That in früherer Zeit, als noch viel kohlensaurer Kalk verordnet wurde, öfters beobachtet worden. Heinrich $v$. B a mberger theilt uns einen solchen Fall mit. Ich glaube aber, dass es genügen dürfte, von Zeit zu Zeit eine energische und grïndliche Entleerung des Darms herbeizuführen, um die Ansammlung grösserer Mengen von Kalk im Darm zu verhindern. Es wird zweckmässig sein, die jetzt so beliebte Cascara sagrada, oder ein grösseres Oelklysma etwa alle 8-16 Tage zu verabreichen. Auch damit ist übrigens gerade nichts neues empfohlen; denn die Oelklysmen hat bei Harngries Fr. Hoffmann vor 200 Jahren verwendet, dessen 'Therapie überhaupt ein glänzendes Muster von Sorgfalt und Ueberlegung ist.

Die Magnesia ist ein sehr altes Mittel: sie war ziemlich in Vergessenheit gerathen, bis sie Brande (und E. Home) im Anfange unseres Jahrhunderts wieder empfahlen. Scudamore verordnete im Gichtanfalle eine Mixtur enthaltend Magnesia usta Magnesia sulfurica, Acetum colchici. Berger hat neuestens wieder eine Magnesiamixtur bei chronischer Gicht sowie im Anfalle empfohlen. Auch die schwer löslichen Verbindungen des Magnesiums können zu Darmsteinbildung Veranlassung geben.

Heute ganz verlassene Mittel sind die Ammoniaksalze. Manche, wie bernsteinsaures Ammoniak, waren im vorigen Jahrhundert beliebte und, wie es scheint, nicht unvernünftige Gichtmittel. Allerdings haben sie alle schlechten Geschmack.

Dafür hat man in den letzten Jahren eine sehr nahe verwandte Gruppe organischer Verbindungen, die Amine herangezogen. Die chemische Industrie hat eine Anzahl solcher Körper auf den Markt gebracht, welche ein sehr bedeutendes Lösungvermögen für Harnsäure besitzen. Es sind:

Piperazin = salzsaures Diäthylendiamin,

Lysidin $=$ Aethylenäthenyldiamin,

Lycetol = weinsaures Dimethyläthylendiamin,

Urotropin = Hexamethylendiamin.

Leider haben sich die Hoffnungen, welche man an diese Substanzen knüpfte, nicht ganz erfüllt. Piperazin, Lysidin und Lycetol haben sich beim Kranken als recht wenig wirksam erwiesen, das Urotropin ertheilt zwar dem Harn eine bedeutende harnsäurelösende Kraft, erzeugt aber oft Blasenbeschwerden, sowie Verdauungsbeschwerden. Es ist jedoch durchaus wahrscheinlich, dass man bei weiterem Suchen noch Aminverbindungen finden dürfte, welche allen Anforderungen genügen. Vorläufig sind diese Präparate sehr theuer.

Als sogenannte "Specificia" könnten wir das salicylsaure Natron und den Harnstoff anführen. Das salicylsaure Natron hat zweifellos innige Beziehnngen zum Harnsäurestoffwechsel, jedoch kennen wir diese heute noch nicht vollständig. Während einige Autoren seine Verwendung empfehlen, weil es die Ansscheidung der Harnsäure vermehrt, befürchten andere, wie Bohland, dass es zum Wachsthum von Tophi und zur Bildung von Concrementen in den Harnwegen Veranlassung geben könnte.

Der Harnstoff wird von Rosenfeld, Klemperer und Leo zur Behandlung der harnsauren Concremente in den Harnwegen - nicht der eigentlichen Gicht - empfohlen. Er ist zweifellos imstande, grosse Harnsäuremengen zu lösen. Dosis 5-10 g per 'Tag.

Ein fast vergessenes "Specificum" ist das Colchicum antumnale. Das Mittel war im ersten Viertel unseres Jahrhunderts sehr angepriesen, nachdem man in ihm den wirksamen Bestandtheil des geheimnissvollen "Eau médicinale" entdeckt hatte, welches der französische Officier Husson verkaufte. Noch Oppolzer wendete es sehr viel an. Eine neue Untersuchung wäre vielleicht am Platze.

Von grosser Wichtigkeit sind die physikalischen Heilmethoden bei der Extremitätengicht. Ausser den warmen Bädern (auch in verschiedenen Thermen) ist es der möglichst frühzeitige Gebrauch der' erkrankt gewesenen Gelenke, worauf Camerer neuerdings hingewiesen hat. Schon van Swieten betonte dies besonders. Er berichtet u. a. von einem Tanzmeister, der für seine hungernde Familie mit schmerzenden Gliedern seine Kunst ausüben musste, dafür aber trotz zahlreicher Anfälle gelenkig blieb. Auch der Werth der Massage ist im vorigen Jahrhundert voll Grosveluor und van Swieten gebülurend liervorgehoben worden, so dass die Neueren nichts wesentliches zil sagen hatten.

Wir haben bis jetzt im allgemeinen von der Gichtbehandlung gesprochen. Es erübrigt die Frage, wie man sich dem einzelnen Gichtanfalle gegenüber zu verhalten habe. Hier stehen sich seit nralter Zeit - seit den Zeiten von Lucian's "Tragopodagra" - zwei Ansichten gegenüber. Die einen empfehlen vollständige Passivität nnd befïrchten, dass jedes Eingreifen, jede Verkürzung des Gichtanfalles, jedes Hinausschieben desselben, ja jede active symptomatische Therapie im Anfalle ein "Zurücktreten" der Gicht zur Folge haben könne. Thr Wahlspruch ist der von Scudamore so getadelte: „Flanell und Geduld“. Die Anderen wenden unbedenklich im Anfalle selbst Alkalien, salicylsaures Natron, Narcotica, Abführmittel, Hydrotherapie (welche Hufeland missbilligte) n. s. w. an. Es ist, wie schon gesagt, noch niclit entschieden, welcho von den Parteien Recht hat.

Wenn wir uns nun iiberlegen, welche Fortschritte wir in der Behandlung durch die eifrige Forschung der letzten Zeit gemacht haben, so müssen wir sagen, dass sie allerdings klein, aber durchans nicht unbedeutend sind. Wir haben ein besseres Verständniss für die Anforderungen bekommen, die wir an die Diätetik zu stellen haben. Wir haben eine neue Gruppe von Verbindungen in den Aminen kennen gelernt, unter welchen wir wirksame Körper mit ernentem Fleisse suchen können. Wir haben im Harnstoff und im salicylsauren Natron körper kennen gelernt, welche in ganz eigenartigen Beziehungen zur Harnsäure stehen. Sind auch hente die nnmittelbar praktisch verwerthbaren nenen Ergebnisse noch gering, so haben wir doch eine Menge neuer Wege gewonnen, anf welchen weiter gearbeitet werden kann. Freilich mïssen wir nns gestehen, dass der Wunsch nach einer specifischen, radicalen Therapie noch ganz und gar nicht erfüllt ist und noch immer der Satz von Sydenham gilt: "Therapeia radicalis et usquequaque perfecta, qua quis etiam a diathesi ad hunc morbum foret liberatus, adhuc in Democriti puteo latet." 